\title{
Ultrasonographic features of the intrinsic foot muscles in patients with and without plantar fasciitis: a novel case-control research study
}

Lorena Canosa-Carro ${ }^{1}$, Daniel López-López ${ }^{2}$, Paula García-Bermejoํㅜㄹ Emmanuel Navarro-Flores ${ }^{3}$, Carmen de Labra ${ }^{4}$, Carlos Romero-Morales ${ }^{1}$

\author{
${ }^{1}$ Department of Physiotherapy, Faculty of Sport Sciences, Universidad Europea \\ de Madrid, Villaviciosa de Odón, Madrid, Spain \\ ${ }^{2}$ Research, Health and Podiatry Unit, Department of Health Sciences, \\ Faculty of Nursing and Podiatry, Universidade da Coruña, Ferrol, Spain \\ ${ }^{3}$ Frailty Research Organized Group (FROG), Department of Nursing, Faculty of Nursing \\ and Podiatry, University of Valencia, Valencia, Spain \\ ${ }^{4}$ Department of Physiotherapy, Medicine and Biomedical Sciences, \\ Universidade da Coruña, Spain
}

Submitted: 13 October 2021; Accepted:15 October 2021

Online publication: 21 November 2021

Arch Med Sci

https://doi.org/10.5114/aoms/143122

Copyright (C) 2021 Termedia \& Banach

\begin{abstract}
Introduction: The aim of the present study was to compare by ultrasound imaging (USI) the thickness and cross-sectional area (CSA) of the flexor hallucis brevis (FHB), flexor digitorum brevis (FDB), abductor hallucis brevis $(\mathrm{AHB})$ and quadratus plantae $(\mathrm{QP})$ muscles between individuals with and without plantar fasciitis (PF).

Material and methods: A case-control study was performed with 64 participants divided into two groups: A, PF group $(n=32)$ and $B$, healthy group $(n=32)$.

Results: USI measurements for FHB CSA $(p=0.035)$ decreased, showing statistically significant differences for the PF group, while the QP CSA $(p=0.40)$ increased, showing statistically significant differences for the PF group with respect to the healthy group. The rest of the intrinsic foot muscles (IFM) did not show statistically significant differences; however in FHB, FDB, QP and AHB thicknesses and FDB CSA showed a slightly decrease for the PF group. Conclusions: USI measurements showed that the CSA of the FHB muscle is reduced in patients with PF while the CSA of the QP muscle is increased in patients with PF.
\end{abstract}

Key words: ultrasonography, musculoskeletal diseases, plantar fascia.

\section{Introduction}

Plantar fasciitis (PF) is the most common cause of heel pain [1]. This condition was described as a degenerative syndrome associated with pain, lack of functionality and stiffness of the plantar fascia [2]. In addition, the calcaneal insertion and mid-foot area were identified as the most common injured locations. Thus, it is frequent to find literature with the terms: neuritis, subcalcaneal bursitis, calcaneal periostitis or heel spur syndrome [3]. Lemont et al. reported that PF episodes could be associated with or without inflammation [4]. Several authors have argued that PF develops due to repeated trauma, therefore considering it
Corresponding author: Carlos Romero PhD Universidad Europea de Madrid, Spain Phone: +34696849724 E-mail: carlos.romero@ unviersidadeuropea.es 
an overuse injury [5]. To date, this condition presents an estimated prevalence of $7 \%$ in the general population, with bilateral involvement reported in $30 \%$ of the affected patients.

The origin of this condition remains unclear despite intrinsic and extrinsic risk factors having been described, such as an excessive body mass index (BMI), reduced ankle dorsiflexion, pes panus, pes cavus, Achilles tendon disturbances, overpronation causing additional stress and weakness of the intrinsic foot muscles and plantar muscles [6] - intrinsic risk factors - and excessive physical activity, low-quality footwear, inadequate surfaces, and walking barefoot [6] - extrinsic factors. Patients with PF experienced severe pain at the first steps in the morning or after non-activity for long periods, such as sleeping or sitting. If these symptoms persist in time, this condition could progress to chronic PF, which leads to an important decrease in quality of life, negative social impact and limited daily activities [7].

Mckeon et al. proposed a concept drawing a parallelism between the intrinsic foot muscles (IFM) and the trunk core muscles, indicating the importance of these small foot muscles for the biomechanics and foot structures (arches) stabilization [8]. The IFM and extrinsic foot muscles (EFM) work in a coordinated manner to provide movement and stability of the three flexible arch structures. In this line, the PF and the IFM - hallucis brevis (AHB), flexor hallucis brevis (FHB), flexor digitorum brevis (FDB) and quadratus plantae (QP) - modify the foot stiffness in order to dissipate external forces or transmit the internal force of the EFM to the foot [9]. Thus, these muscles have been the subject of study for the diagnosis and treatment of foot disorders, such as PF.

Ultrasound imaging (USI) has been employed to evaluate the muscle architecture, such as thickness and cross-sectional area (CSA), related to fascial and muscular disorders. Regarding the foot and ankle area, Wu et al. reported that USI was a reliable method for PF assessment [10]. In the same line, Romero-Morales et al. showed that the thickness of the plantar fascia at the insertion was reduced in individuals with Achilles tendinopathy compared with healthy subjects [11]. Calvo-Lobo et al. reported thickness alterations of the intrinsic plantar muscles in post-stroke survival [12]. Furthermore, Calvo-Lobo et al. observed a reduction in thickness and CSA of the $\mathrm{FHB}$ and $\mathrm{AHB}$ in patients diagnosed with hallux valgus evaluated by USI [13]. Moreover, Angin et al. reported an increase in the PF thickness in patients with pes pannus [14]. Franettovich-Smith et al. showed that the assessment of the IFM by USI had high reliability within and between test sessions and thus was a reliable and clinically feasible method to assess the IFM morphology in weight bearing [15].
To the authors' knowledge, the thickness and CSA of the IFM have not been evaluated in individuals with PF. Thus, the purpose of the present study was to compare the thickness and CSA of the $A H B, F H B$ and $Q P$ in participants with and without PF. Our hypothesis suggests that these muscle structures were altered in patients with PF.

\section{Material and methods}

\section{Design}

An observational study was performed following the Strengthening the Reporting of Observational Studies in Epidemiology (STROBE) specifications from May to August 2021.

\section{Participants}

A total of 64 volunteers from 18 to 55 years were recruited for the present study. The sample was divided in two groups: group A, composed of participants diagnosed with PF $(n=32)$, and group B, composed of healthy participants $(n=32)$. The enrollment of the participants was carried out by a specialized medical doctor with more than 10 years of experience in sport medicine. The group A participants were recruited if they met the following inclusion criteria: heel pain at least 1 month, pain with tenderness on palpation located in the middle of the plantar fascia or at the medial calcaneal tubercle, pain during the first steps in the morning or after non-weight bearing activities $[16,17]$. Exclusion criteria were as follows: fracture, back or lower limb surgery, participants with any systemic disease, pregnancy, infection, plantar orthoses, length discrepancy greater than $1 \mathrm{~cm}$, corticosteroid interventions, and also lower limb disturbances within the last year (e.g. ankle sprain, tendinopathy or tarsal tunnel syndrome) [18].

\section{Sample size calculation}

The sample size calculation was carried out using the $G^{*}$ Power software to measure the difference between the PF and healthy group using the FHB CSA variable of a pilot study $(n=10)$ that was divided into two groups (mean \pm SD): 5 participants with PF $(1.46 \pm 0.29)$ and 5 participants for the healthy group $(1.65 \pm 0.51)$. For the sample size calculation, a power of 0.80 , an $\alpha$ error of 0.05 and an effect size of 0.63 with a one-tailed hypothesis, a power of 0.80 , an $\alpha$ error of 0.05 and an effect size of 0.86 with a one-tailed were employed. In conclusion, a sample of 64 was calculated.

\section{Ethics}

The research ethics committee of Universidad Europea issued a favorable authorization with 
code number CIPI/20/166 for the present study. All the participants signed an informed consent form before study participation. In addition, throughout the study the Helsinki Declaration for human experimentation has been respected.

\section{Ultrasonographic assessment}

For the ultrasonographic assessment an ultrasound system with a high-quality Mindray DC-60 with a 6 to $14 \mathrm{MHz}$ linear transducer (L14-6NE) in B mode was employed. All the USI evaluations were performed by the same clinician with more than 3 years of experience in ultrasound imaging. In accordance with the Mickle et al. procedure, for the AHB, FDB, QP and FHB evaluations, subjects were laid in a supine position with the knee slightly flexed [19]. Crofts et al. reported a high intraand inter-rater reliability agreement for the aforementioned USI protocol [20]. The FHB thickness was measured longitudinally along the $1^{\text {st }}$ meta- tarsal bone at the thickest portion of the muscle (Figure $1 \mathrm{~A}$ ), and after that the transducer was rotated $90^{\circ}$ for the CSA at the same location (Figure 1 B) [19]. The FDB thickness was measured longitudinally along a line from the medial calcaneal tubercle to the third toe at the thickest part of the muscle (Figure $1 \mathrm{C}$ ) and for the CSA the transducer was also rotated $90^{\circ}$ at the same location (Figure 1 D) [19]. In this line, the QP muscle thickness (Figure $1 \mathrm{E}$ ) and CSA (Figure $1 \mathrm{~F}$ ) were evaluated with the transducer in the same place of FDB and located intimately under the FDB muscle. For the AHB assessment the transducer was placed in the middle line between the origin of the muscle on the calcaneal tuberosity and the navicular tuberosity (Figure 2 A). For the AHB CSA the transducer was placed perpendicular to the longitudinal scanning axis of the foot and the anterior plane of the medial malleolus (Figure 2 B) [19]. The final scores were recorded by the mean of
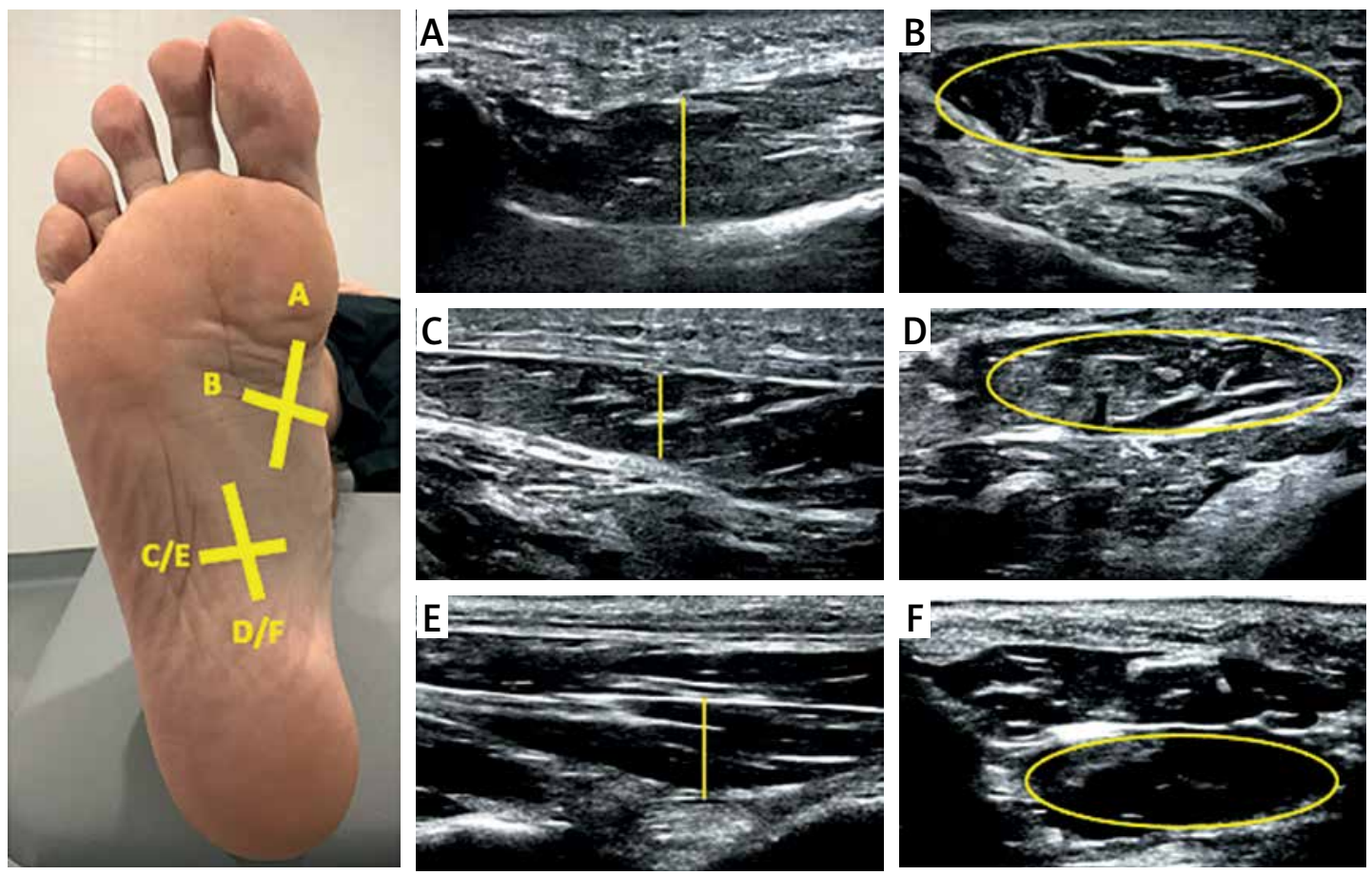

Figure 1. Ultrasound imaging of the FHB, FDB and QP in longitudinal and transversal view
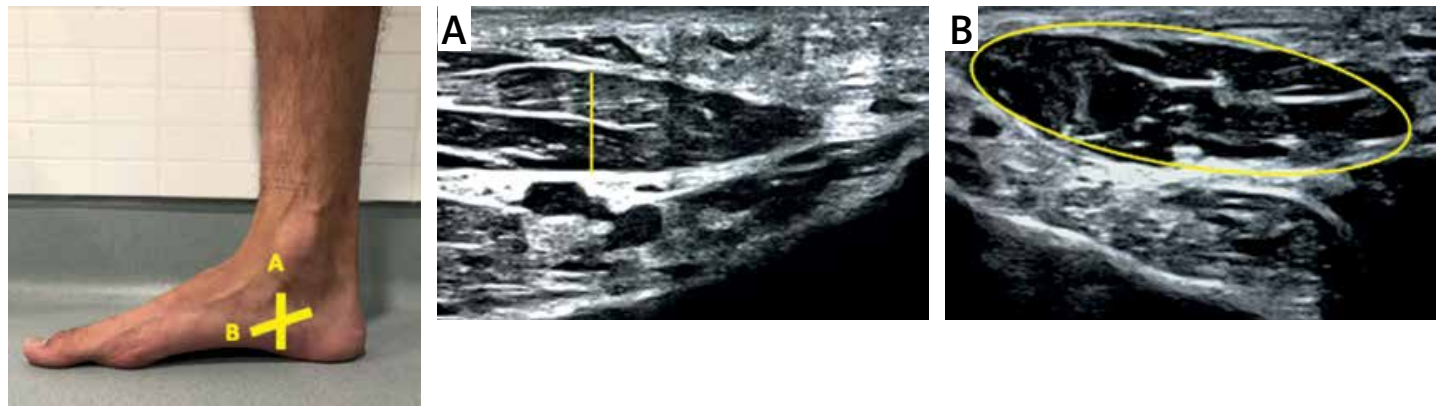

Figure 2. Ultrasound imaging of the AHB in longitudinal and transversal view 
3 repeated values for each measurement with the ImageJ software (Bethesda, MD, USA).

\section{Statistical analysis}

Statistical analysis was performed using IBM SPSS v.22.0 statistical package (SPSS, Inc. Chicago, IL, USA). To check the normality distribution the Kolmogorov-Smirnov test was employed. A descriptive analysis for the sample was performed. A comparative analysis between the PF and the healthy group was carried out. Mean and standard deviation (SD) and Student's $t$ test for independent samples were used for the parametric data. In addition, Levene's test was performed to evaluate the equality of variances. For non-parametric data, median and interquartile range (IR) and the Mann-Whitney $U$ test were performed.

A multivariate analysis was employed using linear regression to predict the influence of the descriptive data on the statistically significant outcome measurements. The dependent variables were FHB and QP CSA. The independent variables were group, sex, age, weight, height, and BMI. An $\alpha$ error of $0.05(95 \% \mathrm{Cl})$ and a desired power of $80 \%$ ( $\beta$ error of 0.2 ) were used.

\section{Results}

According to Table I, sociodemographic data showed significant differences $(p<0.05)$ in age, weight, and body mass index (BMI) between the PF and healthy group. Regarding the IFM, USI measurements for FHB CSA ( $p=0.035)$ decreased, showing statistically significant differences for the PF group, while the QP CSA $(p=0.40)$ increased, showing statistically significant differences for the PF group with respect to the healthy group. The rest of the IFM did not show statistically significant differences; however, in FHB, FDB, QP and AHB thicknesses and FDB CSA a slight decrease for the PF group was observed (Table II).

Regarding the linear regression analysis ( $\mathrm{Ta}$ ble III), the prediction model for FHB CSA $\left(R^{2}=\right.$ $0.122)$ and QP CSA $\left(R^{2}=0.112\right)$ was not determined by the sociodemographic variables.

\section{Discussion}

The results of this research show that individuals with PF have a decrease of FDB CSA and an increase of QP CSA. In addition, for the PF group FHB, $F D B, Q P$ and $A H B$ thicknesses showed a decrease for the PF group with respect to the healthy group. To our knowledge, this is the first study to analyze by USI the differences between PF and healthy participants. Plantar muscles were considered of interest for clinicians and researchers due to the influence in the ankle and foot complex and their relationship with lower limb structures and bio- mechanics, such as the Achilles tendon [14]. Regarding the IFM and lower limb disturbances, such as Achilles tendinopathy, Romero et al. reported an increase of the FDB thickness, as well as FDB and FHB CSA in subjects diagnosed with Achilles tendinopathy assessed by USI [21]. However, the results of the present study showed a decrease for the FDB CSA muscle that might be explained due to these participants presenting a functional deficit in the FDB muscles that had to be compensated by the QP muscle (which increases its CSA). Plantar fascia was described as a structure capable of working with efficiency with greater loads. Therefore, in cases when there are large mechanical load fluctuations, the plantar fascia could be affected, for example, showing changes in the thickness of the plantar fascia in subjects with pes planus [22]. In the same line, Romero-Morales et al. reported that the thickness of the plantar fascia at the insertion was reduced in participants with Achilles tendinopathy [11]. Considering the FHB muscle, Lobo et al. in a previous study observed a decrease in both thickness and CSA variables in participants with hallux valgus [23]. Nevertheless, our results reported similar values with a smaller thickness and CSA in participants with PF. The fact that soft tissue and muscle alterations were described in IFM was closely related to disturbances of the ground reaction forces around the joints of the ankle and foot complex, such PF, hallux valgus, Achilles tendinopathy or pes planus. The present work highlighted the importance of the plantar muscles in the foot and ankle complex; however, it should be considered a very important issue of the foot structure, such as the foot arch, which acts as a passive system which works in a coordinated manner with the IFM muscles to provide stability in weight bearing positions. Based on this criterion, Mickle et al. argued that disturbances of the medial longitudinal arch could be related with PF, foot overpronation or navicular drop situations [19]. The results of the present study suggest that a decrease of the CSA of the FHB muscle could lead to an imbalance in the ankle and foot biomechanics which could be directly related to an increase of the QP CSA in order to compensate the lack of functionality and activity of the FHB muscle, expressed as architecture alterations.

The present study might be a starting point to integrate the use of USI inside the physiotherapist battery test for musculoskeletal condition diagnostics and to track the implementation of training and rehabilitation programs. In this line, the assessment by USI of the intrinsic and extrinsic plantar musculature plays an important role in patients with PF. According to the aforementioned concept, in the Huffer et al. systematic review, the fact of defining the IFM as a therapeutic target, for example with an exercise program, could be of 
Table I. Sociodemographic data of the sample

\begin{tabular}{|lccc|}
\hline Data & Plantar fasciitis $(n=32)$ & Controls $(n=32)$ & $P$-value cases vs. controls \\
\hline Age [years] & $43.00 \pm 11.00^{\dagger}$ & $31.00 \pm 9.50^{\dagger}$ & $0.001^{\star}$ \\
\hline Weight $[\mathrm{kg}]$ & $76.00 \pm 27.00^{\dagger}$ & $73.00 \pm 25.50^{\dagger}$ & $0.028^{\ddagger}$ \\
\hline Height $[\mathrm{m}]$ & $1.71 \pm 6.3^{*}$ & $1.70 \pm 9.68^{\star}$ & $0.465^{\ddagger}$ \\
\hline BMI $\left[\mathrm{kg} / \mathrm{m}^{2}\right]$ & $28.02 \pm 5.56^{*}$ & $24.69 \pm 5.45^{\star}$ & $0.031^{\ddagger}$ \\
\hline
\end{tabular}

$B M I-$ body mass index. ${ }^{*}$ Mean \pm standard deviation (SD) was applied. ${ }^{* *}$ Student's $t$-test for independent samples was performed. ${ }^{\dagger}$ Median \pm interquartile range (IR) was used. ${ }^{\ddagger}$ Mann-Whitney $U$ test was utilized.

Table II. Ultrasound imaging measurements of intrinsic muscle thickness and CSA

\begin{tabular}{|c|c|c|c|}
\hline Distance (unit) & Plantar fasciitis $(n=32)$ & Controls $(n=32)$ & $P$-value \\
\hline FHB CSA $\left[\mathrm{cm}^{2}\right]$ & $1.43 \pm 0.65(1.25-1.72)^{*}$ & $1.76 \pm 0.56(1.56-1.96)^{*}$ & $0.035^{* *}$ \\
\hline FHB Th $[\mathrm{cm}]$ & $1.13 \pm 0.30(1.09-1.26)^{*}$ & $1.17 \pm 0.15(11.11-1.22)^{\star}$ & $0.552^{\star \star}$ \\
\hline FDB CSA $\left[\mathrm{cm}^{2}\right]$ & $1.57 \pm 0.56(1.48-1.76)^{\star}$ & $1.66 \pm 0.34(1.53-1.78)^{*}$ & $0.416^{\star \star}$ \\
\hline FDB Th $[\mathrm{cm}]$ & $0.68 \pm 0.21(0.64-0.77)^{\star}$ & $0.71 \pm 0.14(0.66-0.76)^{*}$ & $0.457^{\star *}$ \\
\hline QP CSA $\left[\mathrm{cm}^{2}\right]$ & $1.23 \pm 0.25(1.00-1.17)^{*}$ & $1.08 \pm 0.33(1.14-1.33)^{*}$ & $0.040^{* *}$ \\
\hline QP Th $[\mathrm{cm}]$ & $0.74 \pm 0.24(0.69-0.84)^{\star}$ & $0.76 \pm 0.18(0.70-0.83)^{*}$ & $0.643^{* \star}$ \\
\hline AHB CSA $\left[\mathrm{cm}^{2}\right]$ & $2.03 \pm 0.72(1.91-2.36)^{*}$ & $2.01 \pm 0.40(1.86-2.15)^{*}$ & $0.841^{\star *}$ \\
\hline AHB Th $[\mathrm{cm}]$ & $1.01 \pm 0.28(0.97-1.11)^{\star}$ & $1.02 \pm 0.24(0.96-1.12)^{*}$ & $0.782^{\star \star}$ \\
\hline
\end{tabular}

Table III. Multivariate predictive analysis for FHB and QP CSA for patients with plantar fasciitis and controls

\begin{tabular}{|c|c|c|c|}
\hline Parameter & Model & $P$-value & Model $R^{2}$ \\
\hline \multirow[t]{6}{*}{ FHB CSA $\left[\mathrm{cm}^{2}\right]$} & 3.430 & & 0.122 \\
\hline & $-0.140 \times \operatorname{Sex}$ & 0.348 & \\
\hline & $-0.125 \times$ Age & 0.077 & \\
\hline & $-0.25 \times$ Weight & 0.344 & \\
\hline & $-0.010 \times$ Height & 0.476 & \\
\hline & $0.084 \times \mathrm{BMI}$ & 0.287 & \\
\hline \multirow[t]{6}{*}{ QP CSA $\left(\mathrm{cm}^{2}\right)$} & 1.351 & & 0.112 \\
\hline & $0.077 \times$ Sex & 0.364 & \\
\hline & $-0.125 \times$ Age & 0.745 & \\
\hline & $-0.25 \times$ Weight & 0.545 & \\
\hline & $-0.010 \times$ Height & 0.961 & \\
\hline & $0.084 \times \mathrm{BMI}$ & 0.513 & \\
\hline
\end{tabular}

FHB - flexor hallucis brevis, $Q P$ - quadratus plantae. ${ }^{*}$ Multiply: Group (control =0; tendinopathy $\left.=1\right)$; Sex $($ women = ; men = 1).

benefit for pain and functionality in patients with $\mathrm{PF}[24]$.

The results of the present study are not intended to provide a cause or explanation about the PF etiology or symptomatology. According to the aforementioned PF, several authors consider the PF a multifactorial musculoskeletal condition [6, 7]. Therefore, the measurement of the IFM by USI as a conventional assessment procedure could be beneficial for the diagnosis and management programs in individuals with PF.

This study has some limitations. First, ultrasonographic measurements were not performed by a researcher blinded to the groups. Second, the $\mathrm{BMI}$ variable was measured by Quetelet's formula reporting differences between groups; thus the results of the present study could be influenced by this [25]. In this line, the weight and BMI differences might be explained by Quetelet's index calculation and also the fact that the heaviest participants could load more and increase their connective tissue work (e.g. muscles or tendons) [26].

Additional studies will be needed to gain a better knowledge about the role of the plantar muscles in individuals with PF. Electromyography or dynamometer tools could clarify the muscle acti- 
vation and its influence on balance and posture. The assessment with $\mathrm{M}$ mode of the muscular activation and inhibition could be useful to extract more information.

In conclusion, USI measurements showed that the CSA of the FHB muscle is reduced in patients with PF while the CSA of the QP muscle is increased in patients with PF. Therefore, the assessment of the IFM by USI could be beneficial for the diagnosis and management programs in individuals with PF.

\section{Conflict of interest}

The authors declare no conflict of interest.

\section{References}

1. Irving DB, Cook JL, Menz HB. Factors associated with chronic plantar heel pain: a systematic review. J Sci Med Sport 2006; 9: 11-4.

2. Rosenbaum AJ, DiPreta JA, Misener D. Plantar heel pain. Med Clin North Am 2014; 98: 339-52.

3. Moyne-Bressand S, Dhieux C, Dousset E, Decherchi P. Effectiveness of foot biomechanical orthoses to relieve patients suffering from plantar fasciitis: is the reduction of pain related to change in neural strategy? Biomed Res Int 2018; 2018: 3594150.

4. Lemont $\mathrm{H}$, Ammirati KM, Usen N. Plantar fasciitis: a degenerative process (fasciosis) without inflammation. J Am Podiatr Med Assoc 2003; 93: 234-7.

5. Choudhary R, Kunal K. Modifiable risk factors of plantar fasciitis in non-athletic patients and proposal of a New Objective Assessment System - RKISP. Rev Bras Ortop 2021; 56: 368-71.

6. Schwartz EN, Su J. Plantar fasciitis: a concise review. Perm J 2014; 18: e105-7.

7. Cotchett M, Rathleff MS, Dilnot M, Landorf KB, Morrissey D, Barton C. Lived experience and attitudes of people with plantar heel pain: a qualitative exploration. J Foot Ankle Res 2020; 13: 12.

8. McKeon PO, Hertel J, Bramble D, Davis I. The foot core system: a new paradigm for understanding intrinsic foot muscle function. Br J Sports Med 2015; 49: 290.

9. Kelly LA, Cresswell AG, Racinais S, Whiteley R, Lichtwark G. Intrinsic foot muscles have the capacity to control deformation of the longitudinal arch. J R Soc Interface 2014; 11: 20131188.

10. Wu J, Zhang YZ, Gao Y, Luo TY. Assessment the reliability of ultrasonography in the imaging of the plantar fascia: a comparative study. BMC Med Imaging 2019; 19: 1-8.

11. Romero-Morales C, Martín-Llantino PJ, Calvo-Lobo C, et al. Ultrasonography features of the plantar fascia complex in patients with chronic non-insertional achilles tendinopathy: a case-control study. Sensors 2019; 19 2052.

12. Calvo-Lobo C, Useros-Olmo Al, Almazán-Polo J, et al. Quantitative ultrasound imaging pixel analysis of the intrinsic plantar muscle tissue between hemiparesis and contralateral feet in post-stroke patients. Int J Environ Res Public Health 2018; 15: 2519.

13. Lobo CC, Marín AG, Sanz DR, et al. Ultrasound evaluation of intrinsic plantar muscles and fascia in hallux valgus: a case-control study. Medicine 2016; 95: e5243.
14. Angin S, Crofts G, Mickle KJ, Nester CJ. Ultrasound evaluation of foot muscles and plantar fascia in pes planus. Gait Posture 2014; 40: 48-52.

15. Franettovich Smith MM, Hides JA, Hodges PW, Collins NJ. Intrinsic foot muscle size can be measured reliably in weight bearing using ultrasound imaging. Gait Posture 2019; 68: 369-74.

16. Thong-On S, Bovonsunthonchai S, Vachalathiti R, Intiravoranont W, Suwannarat S, Smith R. Effects of strengthening and stretching exercises on the temporospatial gait parameters in patients with plantar fasciitis: a randomized controlled trial. Ann Rehabil Med 2019; 43: 662-76.

17. Renan-Ordine R, Alburquerque-Sendín F, De Souza DPR, Cleland JA, Fernández-De-Las-Penas C. Effectiveness of myofascial trigger point manual therapy combined with a self-stretching protocol for the management of plantar heel pain: a randomized controlled trial. J Orthop Sports Phys Ther 2011; 41: 43-50.

18. Dunning J, Butts R, Henry N, et al. Electrical dry needling as an adjunct to exercise, manual therapy and ultrasound for plantar fasciitis: a multi-center randomized clinical trial. PLoS One 2018; 13: e0205405.

19. Mickle KJ, Angin S, Crofts G, Nester CJ. Effects of age on strength and morphology of toe flexor muscles. J Orthop Sports Phys Ther 2016; 46: 1065-70.

20. Crofts G, Angin S, Mickle KJ, Hill S, Nester CJ. Reliability of ultrasound for measurement of selected foot structures. Gait Posture 2014; 39: 35-9.

21. Romero-Morales C, Martin-Llantino PJ, Calvo-Lobo C, et al. Intrinsic foot muscles morphological modifications in patients with Achilles tendinopathy: a novel case-control research study. Phys Ther Sport 2019; 40: 208-12.

22. Huang YC, Wang LY, Wang HC, Chang KL, Leong CP. The relationship between the flexible flatfoot and plantar fasciitis: ultrasonographic evaluation. Chang Gung Med J 2004; 27: 443-8.

23. Lobo CC, Marin AG, Sanz DR, et al. Ultrasound evaluation of intrinsic plantar muscles and fascia in hallux valgus: a case-control study. Medicine 2016; 95: e5243.

24. Huffer D, Hing W, Newton R, Clair M. Strength training for plantar fasciitis and the intrinsic foot musculature: a systematic review. Phys Ther Sport 2017; 24: 44-52.

25. Garrow JS, Webster J. Quetelet's index (W/H2) as a measure of fatness. Int J Obes 1985; 9: 147-53.

26. Foley D, Guralnik JM, Leveille SG, et al. Muscle strength and body mass index as long-term predictors of mortality in initially healthy men. J Gerontol Ser A 2000; 55: M168-73. 